\title{
Circular RNAs: new genetic tools in melanoma
}

Jamal Halajzadeh1, Elaheh Amirani², Hamed Mirzaei**,2, Rana Shafabakhsh², Seyyed Mehdi Mirhashemi ${ }^{3}$, Mehran Sharifi ${ }^{4}$, Bahman Yousefi ${ }^{5}$, Mohammad Ali Mansournia ${ }^{6}$ \& Zatollah Asemi*,2

${ }^{1}$ Department of Biochemistry \& Nutrition, Research Center for Evidence-Based Health Management, Maragheh University of Medical Science, Maragheh, Iran

${ }^{2}$ Research Center for Biochemistry \& Nutrition in Metabolic Diseases, Kashan University of Medical Sciences, Kashan, Iran

${ }^{3}$ Metabolic Diseases Research Center, Research Institute for Prevention of Non-Communicable Diseases, Qazvin University of Medical Sciences, Qazvin, Iran

${ }^{4}$ Department of Hematology \& Oncology, School of Medicine, Isfahan University of Medical Sciences, Isfahan, Iran

${ }^{5}$ Stem Cell Research Center, Tabriz University of Medical Sciences, Tabriz, Iran

${ }^{6}$ Department of Epidemiology \& Biostatistics, School of Public Health, Tehran University of Medical Sciences, Tehran, Iran

*Author for correspondence: Tel.: +98-31-55463378; Fax: +98-31-55463377; asemi_r@yahoo.com

**Author for correspondence: h.mirzaei2002@gmail.com

Melanoma is the most lethal form of skin cancer. New technologies have resulted in major advances in the diagnosis and treatment of melanoma and other cancer types. Recently, some studies have investigated the role of circular RNAs (circRNAs) in different cancers. CircRNAs are a member of long noncoding RNA family mainly formed through back-splicing and have a closed loop structure. These molecules affect several biological and oncogenic cascades in diverse ways via acing as microRNA sponge, interacting with RNA-binding proteins and acting as a transcription regulator. In this review, we made an insight into the impact of circRNA dysregulation in the melanoma tumorigenesis based on the presented evidences.

First draft submitted: 4 December 2019; Accepted for publication: 23 March 2020; Published online: TBC

Keywords: biomarker • circular RNAs • genetic regulation • melanoma

Melanoma, the cancerous growth of melanocytes, is the most aggressive form of all skin cancers [1]. This cancer is more common in the men, with worse survival rate than women $[2,3]$. Recently, its incidence, morbidity and mortality are increasing worldwide. The American Cancer Society estimated that in 2019 melanoma was the fourth most common cancer in men and the fifth most common cancer in women [4]. There are multiple distinct categories of melanocytic neoplasms which are different in cell of origin, role of ultraviolet radiation, clinical and histological indications, mutation pattern, predisposing germ line alterations and sites of metastasis [5]. Based on the primary site of neoplasm and morphologic aspects of the early growth phase, melanoma divided into four main types: superficial spreading melanoma (50-75\%), nodular melanoma (15-35\%), lentigo malignant melanoma (5-15\%) and acrallentiginous melanoma (5-10\%) [6,7].

Recent investigations and new technologies have resulted in major advances in the diagnosis and treatment of this cancer. In addition to conventional therapies for melanoma such as surgical intervention and radiation therapy, other treatment options including chemotherapy, immunotherapy, genetically targeted therapy and nanotechnology have been used [8-10]. Although melanoma is treatable in its early stages, it is still a therapeutic challenge and advanced malignant melanoma carries a poor prognosis. Patients with stages II and III have a 10-year survival rate of 77 and $69 \%$, respectively [11]. One of the main problems in the treatment of melanoma is low response rate to the present treatment modalities due to inherent resistance of melanoma cells to chemotherapeutic agents [12]. Melanoma arises from a complex interaction between environmental and genetic factors. A well-established risk factor for melanoma development is exposure to ultraviolet radiation, which induces cellular stress signaling, DNA damage and disrupts DNA repair systems [13,14]. Multiple pathogenic mutations in melanoma affect genes involved in key signaling pathways that control proliferation (mitogen-activated protein kinase [MAPK], NF1, NRAS, and BRAF)

Future Medicine 